\title{
Clinical Course of COVID-19 in Identical Twins
}

\author{
Mishita Goel $^{\mathrm{a}, \mathrm{b}}$, Victoria Gonzalez ${ }^{\mathrm{a}}$, Reina Badran ${ }^{\mathrm{a}}$, Vesna Tegeltija ${ }^{\mathrm{a}}$
}

\begin{abstract}
The rapid outbreak of coronavirus disease 19 (COVID-19), caused by severe acute respiratory syndrome coronavirus 2 (SARS-CoV-2), has led to infection with variable clinical presentations and a wide clinical spectrum. The disease was first reported in Wuhan, China in 2019 and has rapidly spread worldwide. Despite reports of dynamic changes in disease progression, clinical predictors of disease severity have been difficult to identify. The following case describing identical twins with laboratory confirmed COVID-19 who had very different disease courses. These patients resided in the same home and shared many of the same comorbidities, including type 2 diabetes mellitus, hypertension and morbid obesity. Although twin 1 had higher inflammatory markers, white blood cell (WBC) and an arguably more complicated medical history in comparison to their identical twin, the patient experienced a milder and shorter disease course. This case highlights the need for identifying proper disease markers and predictors early in the clinical course in order to direct future management guidelines and timely treatment.
\end{abstract}

Keywords: Clinical course; COVID-19; Identical twins

\section{Introduction}

In December 2019, an outbreak of pneumonia was reported in Wuhan, Hubei Province, China [1]. By January 2020, the causative organism was identified to be a novel RNA virus, named severe acute respiratory syndrome coronavirus 2 (SARS-CoV-2). The virus causes a respiratory illness, described as coronavirus disease 19 (COVID-19) [2]. Cases of COVID-19 rapidly increased worldwide, quickly reaching pandemic level.

The clinical spectrum of COVID-19 ranges from asymptomatic infection to respiratory failure and death. Worldwide, the medical community is attempting to better understand all

Manuscript submitted June 20, 2020, accepted July 8, 2020

Published online August 28, 2020

aDepartment of Internal Medicine, WSU/Ascension Providence Rochester Hospital, Rochester, MI, USA

${ }^{b}$ Corresponding Author: Mishita Goel, Department of Internal Medicine, WSU/Ascension Providence Rochester Hospital, 1101 W University Dr, Rochester, MI 48307, USA. Email: goelmishita@gmail.com

doi: https://doi.org/10.14740/jmc3536 aspects of this novel virus. One key interest of research is the wide variability of disease course, ranging from no symptoms to death. In order to improve management strategies, it is important to understand indicators of disease severity. Here we present a case of identical twin sisters who were admitted to the hospital with respiratory symptoms of similar duration and were found to have laboratory confirmed COVID-19 pneumonia. It is assumed that both patients acquired the virus from the same source and the same strain; nevertheless, they had a very different clinical course and outcome.

\section{Case Reports}

\section{Case 1}

This is a 35-year-old, morbidly obese woman (body mass index (BMI) 44) with a history of uncontrolled type 2 diabetes mellitus (last A1c 10.1\%), hypertension (not on any antihypertensive medication) and asthma who presented with shortness of breath, cough and fever. Symptoms started 4 days prior and shortness of breath worsened, prompting an emergency department (ED) visit. The patient also endorsed decreased sense of smell and poor appetite. She worked at a daycare facility and did report exposure to a COVID-19-positive coworker.

She was evaluated in the ED. Chest X-ray and labs were obtained. Testing for COVID-19 was sent. The patient had a temperature of $38.1{ }^{\circ} \mathrm{C}$, blood pressure (BP) $140 / 92$, heart rate (HR) $80 / \mathrm{min}$ and respiratory rate (RR) $18 / \mathrm{min}$. She was saturating at $92 \%$ on $2 \mathrm{~L}$ of oxygen via nasal cannula. Her lungs were clear to auscultation.

Chest X-ray showed patchy infiltrates in the left perihilar region and right lower lung. Labs revealed hyperglycemia with a glucose of $150 \mathrm{mg} / \mathrm{dL}$ and elevated inflammatory markers as noted in Table 1. The patient was admitted with suspected COVID-19 diagnosis and placed in isolation while awaiting COVID-19 testing.

Repeat chest X-ray showed worsening ground glass opacities with focal infiltrates in right upper and left lower chest. The patient remained in the hospital for 6 days. Throughout her stay, oxygen requirement was $2-3 \mathrm{~L}$ by nasal cannula. She received treatment with 5 days of azithromycin $(500 \mathrm{mg})$, and 3 days of hydroxychloroquine $(400 \mathrm{mg})$. Her repeat labs revealed a stable pattern with a decrease in Creactive protein (CRP) (Figs. 1,2). She was discharged home in stable condition with advice to continue self-quarantine and an education on appropriate precautions was provided. 
Table 1. Initial Lab Results on Admission

\begin{tabular}{|c|c|c|}
\hline & Twin 1 & Twin 2 \\
\hline Glucose (mg/dL) & 150 & 102 \\
\hline Sodium (mEq/L) & 135 & 136 \\
\hline Chloride (mEq/L) & 97 & 98 \\
\hline Potassium (mEq/L) & 3.9 & 4.2 \\
\hline Bicarbonate $(\mathrm{mEq} / \mathrm{L})$ & 24.2 & 21.5 \\
\hline BUN/creatinine & $11 / 0.7$ & $15 / 1.0$ \\
\hline Hemoglobin (g/dL) & 12.4 & 12.1 \\
\hline WBCs $\left(10^{3} / \mu \mathrm{L}\right)$ & 4.8 & 4.9 \\
\hline Neutrophil\% & 52 & 57 \\
\hline Lymphocyte\% & 35 & 35 \\
\hline Platelets $\left(10^{3} / \mu \mathrm{L}\right)$ & 154 & 156 \\
\hline Calcium (mg/dL) & 9 & 8.9 \\
\hline Albumin (g/dL) & 3.4 & 3.6 \\
\hline $\mathrm{BNP}(\mathrm{pg} / \mathrm{mL})$ & $<5$ & 10 \\
\hline $\mathrm{CRP}(\mathrm{mg} / \mathrm{dL})$ & 11.77 & 3.66 \\
\hline $\operatorname{ESR}(\mathrm{mm} / \mathrm{h})$ & 87 & 70 \\
\hline Ferritin (ng/mL) & 184 & 54 \\
\hline $\mathrm{LDH}(\mathrm{U} / \mathrm{L})$ & 312 & 236 \\
\hline Procalcitonin (ng/mL) & 0.05 & 0.12 \\
\hline $\operatorname{ALP}(\mathrm{U} / \mathrm{L})$ & 83 & 73 \\
\hline $\operatorname{AST}(\mathrm{U} / \mathrm{L})$ & 23 & 22 \\
\hline $\operatorname{ALT}(\mathrm{U} / \mathrm{L})$ & 13 & 10 \\
\hline Bilirubin (mg/dL) & 0.26 & 0.14 \\
\hline
\end{tabular}

BUN: blood urea nitrogen; WBCs: white blood cells; BNP: brain natriuretic peptide; CRP: C-reactive protein; ESR: erythrocyte sedimentation rate; LDH: lactate dehydrogenase; ALP: alkaline phosphatase; AST: aspartate transaminase; ALT: alanine aminotransferase.

\section{Case 2}

This is a 35-year-old, morbidly obese woman (BMI 44) with a history of well controlled type 2 diabetes mellitus (last A1c 6.4\%) and hypertension (not on any antihypertensive medication) who was admitted to the hospital on the same day as her sister (twin 1) with chief complaint of worsening dyspnea. The patient also reported subjective fevers that started around the same time as her twin sister. The patient lives with her twin sister (twin 1) who had similar symptoms after a close contact with a COVID-19 positive coworker.

On admission, the patient had a temperature of $38.7^{\circ} \mathrm{C}$, BP 123/82, HR 98/min and RR 16/min. She was saturating at $91 \%$ on room air. Her lungs were clear to auscultation. Chest X-ray showed bilateral patchy ground glass opacities. Lab values revealed mild inflammatory marker changes; otherwise were stable on presentation (Table 1). The patient was placed on (4 L) oxygen (via nasal cannula) and admitted to the medical floor with suspected COVID-19 diagnosis while COVID-19 testing was pending. Treatment for bacterial pneumonia was also initiated with IV Ceftriaxone and Azithromycin. She was treated with ceftriaxone and azithromycin for pneumonia.

On day 4 of hospitalization, the patient suddenly developed extreme dyspnea and had increased work of breathing. Subsequently, her oxygen requirement significantly increased from $4 \mathrm{~L}$ to $12 \mathrm{~L}$ nasal cannula (NC) with $\mathrm{SpO}_{2}$ improving to $90-92 \%$. The patient also developed high fevers reaching $40{ }^{\circ} \mathrm{C}$. Repeat Chest X-ray showed interval increased confluence of bilateral infiltrates. Treatment with hydroxychloroquine, zinc $220 \mathrm{mg}$ and prednisone $60 \mathrm{mg}$ daily was initiated in addition to above noted antibiotics. The patient was transferred to the intensive care unit (ICU) for closer monitoring and further management of respiratory failure. Her repeat labs showed an increase in lactate dehydrogenase (LDH), white blood cell (WBC) and inflammatory markers including CRP and erythrocyte sedimentation rate (ESR). Percentage of lymphocytes decreased, and percentage of neutrophils in-
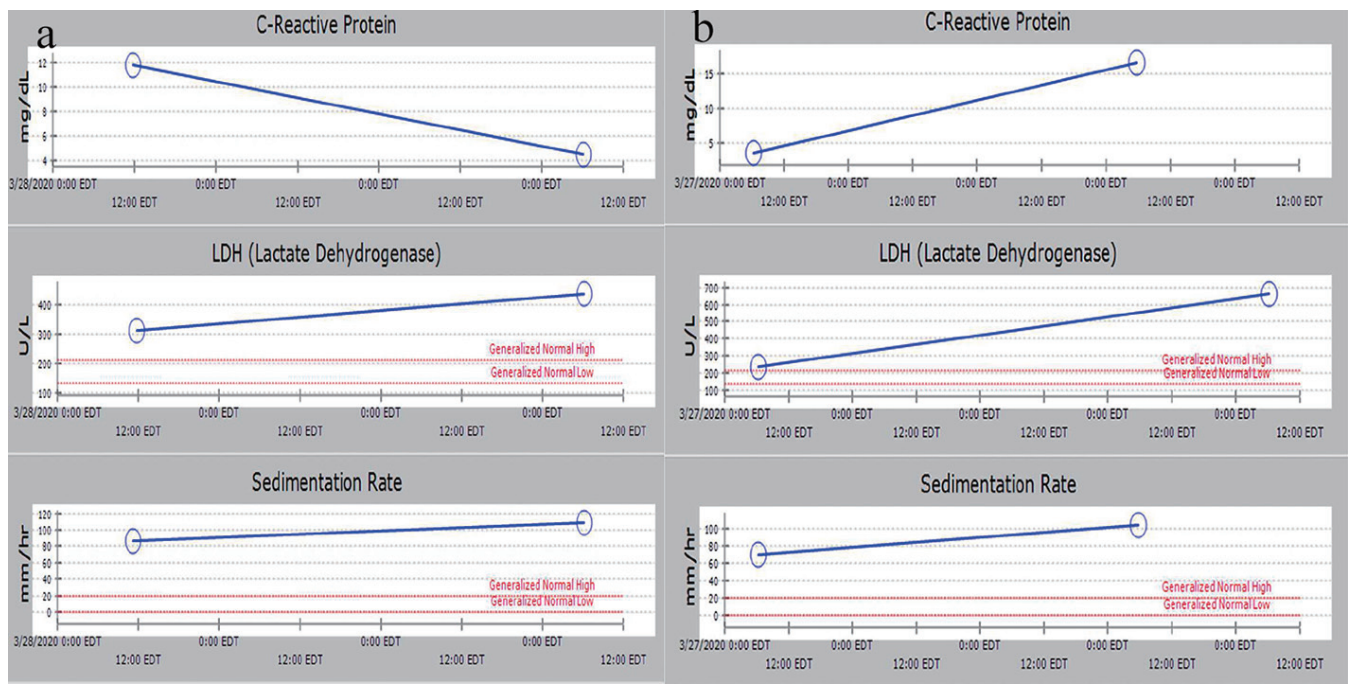

Figure 1. Trend of inflammatory markers: (a) twin 1 and (b) twin 2. 


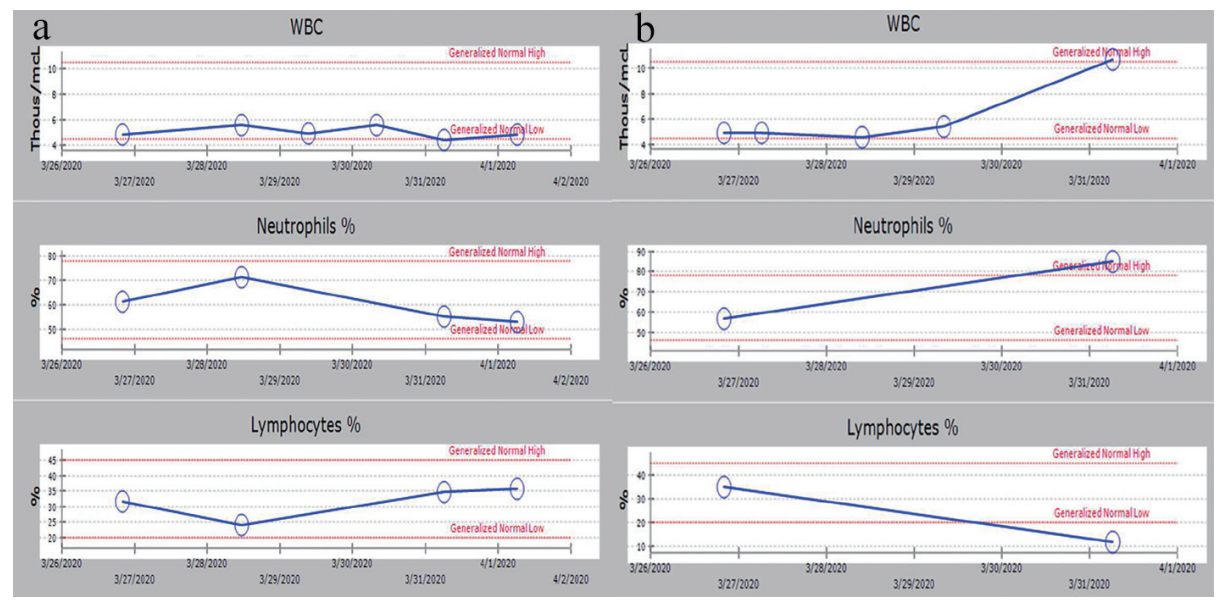

Figure 2. Trend of WBC count, lymphocyte\% and neutrophil\%: (a) twin 1 and (b) twin 2. WBC: white blood cell.

creased (Figs. 1, 2).

In the ICU, patient was intubated due to worsening hypoxic respiratory failure. She was kept in prone position overnight and was started on neuromuscular blocking agents. She was on bilevel ventilator settings of positive end-expiratory pressure (PEEP) 40/18, $\mathrm{FiO}_{2} 100 \%$, T1 2.25 s, and I:E 3:1. Patient was given a dose of intravenous (IV) Lasix $20 \mathrm{mg}$ following which she developed acute kidney injury. Her creatinine worsened and urine output declined to $0.2 \mathrm{cc} / \mathrm{kg} / \mathrm{h}$. She also became hypotensive. Fluid resuscitation was given but eventually patient had to be started on vasopressors due to persistent shock. The patient had multiple episodes of desaturation despite being on $\mathrm{FiO}_{2}$ of $100 \%$. After discussion with the family, it was decided to transfer her to a tertiary care center for extracorporeal membrane oxygenation (ECMO) and availability of renal replacement therapy if needed. Patient was transferred in critical condition and reached the facility. The team followed up with a phone call and discovered that the patient was treated at the tertiary center where she remained hospitalized for weeks. She was eventually weaned off the ventilator, extubated and discharged home.

\section{Discussion}

The rapid emergence of COVID-19 infection has caused thousands of deaths worldwide [3]. This novel virus can cause infection with variable clinical presentations ranging from no symptoms to acute respiratory failure and death. Although many research articles are reporting clinical characteristics, little is known about indicators of these clinical characteristics and dynamic changes during disease progression. Identifying clinical predictors of disease severity and outcome is important to help guide clinical care and allocate resources. The patient cases described above are that of identical twins with laboratory-confirmed COVID-19 who had very different disease courses. We assume that twin 1 acquired the infection at work and infected twin 2 based on the reported COVID-19-positive contact. The identical twin patients live together and had a similar timeline of symptoms and ED presentation. Furthermore, the identical twins both have a history of diabetes, hypertension and morbid obesity with the same BMI of 44. Twin 1 additionally has asthma and her diabetes is uncontrolled. We reviewed the symptoms, oxygen requirement, chest X-ray findings and lab values of both patients. Next, we analyzed the changes in their peripheral blood to identify lab markers and recognize an association, if any, with disease severity and outcome.

Table 1 compares admission lab findings between the identical twin patients. Lab values are similar. Twin 1 had slightly higher glucose readings on admission. Twin 1, on admission, had higher inflammatory markers, ESR, CRP, ferritin and LDH. Based on these results, her direct exposure, uncontrolled diabetes, and asthma, one would suspect that twin 1 would have a more complicated disease progress when compared to twin 2. However, as noted in the case, twin 1 had a milder course and was discharged home in stable condition in a few days. Twin 2, who we assume contracted the virus from her sister, had better controlled diabetes and no asthma history. Her disease course was more complicated, and she required ICU care and intubation.

Figures 1 and 2 demonstrate comparison of labs between the two patients during the hospitalization. As Figure $1 \mathrm{dem}-$ onstrates, both patients had an increase in LDH and ESR. However, CRP decreased in twin 1 and increased in twin 2, who had a more severe clinical course. Figure 2 demonstrates that WBC count and neutrophil percentage increased for twin 2 while lymphocyte percentage decreased. Conversely, twin 1 had stable WBC counts and an increase in lymphocyte percentage as she clinically improved. In conclusion, data suggest that a rising CRP, rising WBC and a decreasing lymphocyte percentage may suggest worse clinical outcomes.

Previous studies have shown lymphopenia to be a predictor of prognosis in COVID-19 patients and that lymphocyte percentage can be independently used to classify disease severity in addition to other auxiliary indicators [4]. The twin patients had the same lymphocyte percentages on presentation. Twin 1, who recovered quickly with only supportive care, was observed to have more than $20 \%$ lymphocytes throughout her stay, while twin 2 was found to have a decrease in the lymphocyte percentage (as low as 11\%) throughout the stay. This 
was in conjunction with the findings of study by Tan et al [4] where patients with more than $20 \%$ lymphocytes were classified as moderate and recovered quickly. Multiple mechanisms have been proposed to explain lymphopenia in COVID-19 which include direct infection and destruction via angiotensin I converting enzyme 2 (ACE-2) receptor attachment on lymphocytes, apoptosis due to cytokine storm, inhibition of proliferation due to lactic acidemia, etc.

Studies also reported that ICU admission in patients with COVID-19 pneumonia is associated with high serum neutrophil count and CRP levels [5]. The levels of CRP, ESR and LDH in twin 2 did trend upwards with worsening clinical status. These findings can be explained by COVID-19 progression to third and most severe stage of illness, systemic hyperinflammation. During this stage, all inflammatory markers are elevated [6]. For unclear reasons, similar LDH and ESR trend was observed in the twin patients. For twin 1, CRP levels trended down, correlating with clinical improvement. Further data review may assist in establishing a statistically significant correlation between inflammatory changes leading to disease progression.

A study of 150 patients from Wuhan showed that increased number of comorbid illnesses is associated with increased mortality [7]. Even the report of 72,314 COVID-19 cases as published by Chinese Center for Disease Control and Prevention (CDC) showed association of diabetes mellitus with increased mortality [8]. Both the twin patients were morbidly obese and had hypertension and type 2 diabetes mellitus. Even though twin 2 had better control of diabetes, she was seen to have severe infection and quick clinical deterioration. A meta-analysis of 33 studies showed that the presence of diabetes itself was associated with severe COVID-19 infection and increased mortality [9].

Treatment strategies to decrease inflammation, early in the course, when the inflammatory markers are trending up, may aid in decreasing inflammation and improving outcomes. These indicators might be helpful in guiding clinical care even before clinical worsening and in future can be included in management guidelines to direct treatment at early stages.

\section{Acknowledgments}

None to declare.

\section{Financial Disclosure}

None to declare.

\section{Conflict of Interest}

None to declare.

\section{Informed Consent}

Written consent could not be obtained since patient had to be transferred to other facility; verbal consent was obtained during follow-up call.

\section{Author Contributions}

All authors have contributed significantly to drafting and reviewing the manuscript, making graphs and most importantly, management of these patients.

\section{Data Availability}

The authors declare that data supporting the findings of this study are available within the article.

\section{References}

1. Phelan AL, Katz R, Gostin LO. The novel coronavirus originating in Wuhan, China: Challenges for Global Health Governance. JAMA. 2020;323(8):709-710.

2. Zhu N, Zhang D, Wang W, Li X, Yang B, Song J, Zhao X, et al. A novel coronavirus from patients with pneumonia in China, 2019. N Engl J Med. 2020;382(8):727-733.

3. Mahase E. Coronavirus covid-19 has killed more people than SARS and MERS combined, despite lower case fatality rate. BMJ. 2020;368:m641.

4. Tan L, Wang Q, Zhang D, Ding J, Huang Q, Tang YQ, Wang Q, et al. Lymphopenia predicts disease severity of COVID-19: a descriptive and predictive study. Signal Transduct Target Ther. 2020;5(1):33.

5. Ran J, Dai B, Wang D, Li XM. Clinical predictors of severity based on an analysis of data of COVID-19 pneumonia. EBioMedicine. 2020.

6. Siddiqi HK, Mehra MR. COVID-19 illness in native and immunosuppressed states: A clinical-therapeutic staging proposal. J Heart Lung Transplant. 2020;39(5):405-407.

7. Ruan Q, Yang K, Wang W, Jiang L, Song J. Correction to: Clinical predictors of mortality due to COVID-19 based on an analysis of data of 150 patients from Wuhan, China. Intensive Care Med. 2020;46(6):1294-1297.

8. Wu Z, McGoogan JM. Characteristics of and important lessons from the coronavirus disease 2019 (COVID-19) outbreak in China: summary of a report of 72314 cases from the Chinese Center for Disease Control and Prevention. JAMA. 2020;323(13):1239-1242.

9. Kumar A, Arora A, Sharma P, Anikhindi SA, Bansal N, Singla V, Khare S, et al. Is diabetes mellitus associated with mortality and severity of COVID-19? A meta-analysis. Diabetes Metab Syndr. 2020;14(4):535-545. 